\title{
Papillary Muscle Segmentation from a Multi-Atlas Database: A Feasibility Study
}

\author{
Benedetta Biffi ${ }^{1}$, Maria A. Zuluaga ${ }^{2}$, Sébastien Ourselin ${ }^{2}$, \\ Andrew M. Taylor ${ }^{3,4}$, and Silvia Schievano ${ }^{3,4}$ \\ ${ }^{1}$ UCL Dept of Medical Physics and Biomedical Engineering, University College \\ London, UK \\ 2 Translational Imaging Group, Centre for Medical Image Computing, University \\ College London, UK \\ ${ }^{3}$ UCL Institute of Cardiovascular Science, University College London, UK \\ ${ }^{4}$ Great Ormond Street Hospital for Children, London, UK
}

\begin{abstract}
Mitral valve regurgitation replacement could be benefited by the use of pre-surgical 3D models that would allow the clinician to better understand the patient's structural and functional information before surgery. As no single image modality can provide all this information, it is necessary to fuse 3D transthoracic echo (TTE) and magnetic resonance imaging (MRI) to obtain a complete model. The registration of these two modalities, within the context of cardiac imaging, is a challenging task that requires manual intervention or the use of anatomical landmarks that can drive the registration. Within the context of mitral valve planning, the papillary muscles represent an ideal landmark set as they can be clearly identified in both modalities. However, to date little work has addressed the problem of papillary muscle segmentation. In this paper, we apply an atlas-based segmentation method for the automatic extraction of papillary muscles from MRI. Results show that a good quality segmentation (Dice score $0.67 \pm 0.13$ ) can be achieved within the straightforward pipeline provided by this approach. Hence, our atlas-based segmentation method could represent the first key step towards a novel TTE-MRI fusion algorithm.
\end{abstract}

\section{Introduction}

Mitral valve (MV) regurgitation is a common form of valvular abnormality which requires treatment or replacement through invasive open-heart surgery, with considerable risk of significant morbidity and mortality [1]. Thus, there is an increased demand for minimally invasive techniques, such as sutureless and/or transcatheter MV replacement. These procedures have already been developed and successfully used for the replacement of aortic and pulmonary valves, but are not yet available for the MV, as its anatomy is complex, with a non-uniform geometry that relies on several inter-related components for its function: the annulus, the leaflets, the chordae tendineae, and the supporting papillary muscles. Clinically, 3D transthoracic echo is the principal technique used for MV 

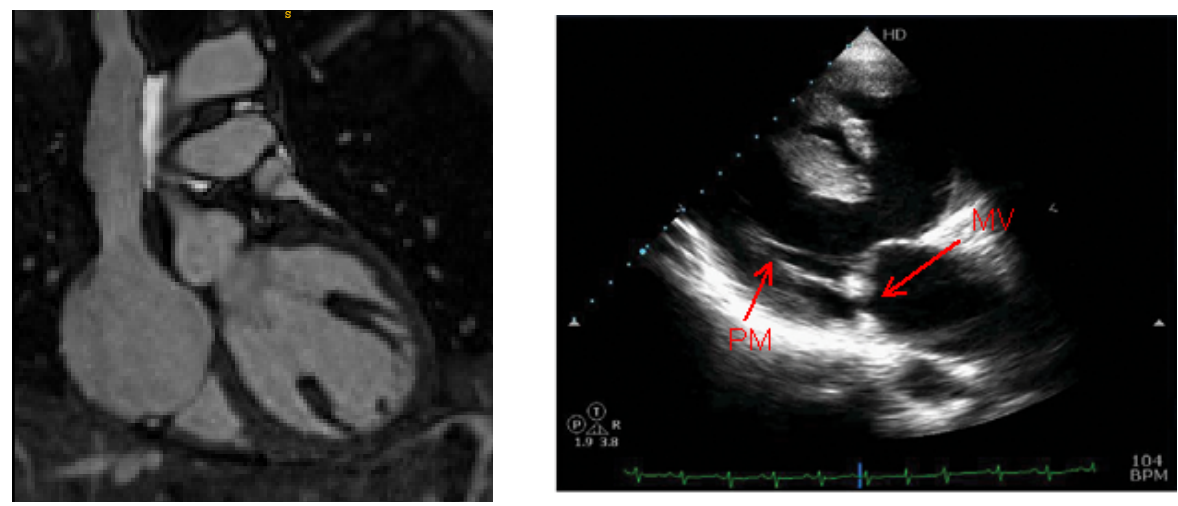

Fig. 1. Papillary muscles highlighted in both image modalities. a) Coronal view of cardiac whole-heart MRI. The papillary muscles are higlighted by the red arrows. Details of the chordae and the MV leaflets are also visible. b) Parasternal long-axis view of 3D-TTE.

assessment, offering real-time structural and functional information. With the advances of ultrasound probes, high-resolution, full-volume imaging and quantification of the morphology of the entire MV apparatus have become feasible. Magnetic resonance imaging (MRI) is the reference standard for the measurement of ventricular and atrial size, geometry and function. By combining these two imaging modalities, it would be possible to fill in information missing from TTE or MRI alone to create a detailed 3D model of the left heart and MV.

A pre-requisite for the creation of a fused model is the alignment of both image modalities. This is a big challenge as the $3 \mathrm{D}$ registration of TTE to MRI remains to be an open problem. To date, most existing approaches for echo to MRI (or CT) registration have been used in neurosurgical applications [2, 3]. Cardiac echo-MR registration has been addressed to a lesser extent [4-6]. Currently developed methods require either some manual initial registration [4, 5] or the definition of landmarks [6] in both images that can guide the registration procedure. Landmark-based approaches [6] for TTE-MRI registration represent a good trade-off between accuracy, ease of use and computational time. However, due to the limited field of view (FOV) of TTE it is required to define landmarks that are specific to the problem (i.e. anatomical region) that is addressed.

Within the context of mitral valve planning, the papillary muscles (PM) represent an ideal landmark set as they can be clearly identified in both TTE and MRI. (Fig. 1). With the final aim of fusing 3D TTE and MRI for MV pre-surgical planning, in this work we first address the problem of accurately extracting the PM from MR images.

While the segmentation of the main chambers of the heart has been widely addressed [7], little work has focused on the extraction of smaller structures such as the PM. Spreeuwers et al. [8] were the first to tackle the problem through a 2D region-based approach. More recently, Gao et al. [9] addressed the problem 
through a topological method that tried to restore missing structures from an initial segmentation using high resolution CT. Other methods in the literature, have extracted the PM along with the left ventricle but, not as a separate structure. Despite the popularity of atlas-based methods in the segmentation of the heart $[10,7]$, none of the existing works have tried to use this type of framework for the segmentation of the PM. This could be explained by the complexity, shape, size and position variability of these. The final aim of this work is to demonstrate, through a validation study, that atlas-based approaches are well suited for PM segmentation.

The remaining of this paper is organised as follows: Section 2 describes the data and the specific methods used for this study. Section 3 shows the results. Finally, a discussion on the obtained results and a conclusion are presented in Section 4.

\section{Materials and Methods}

In this section we describe the data, the motivation for an atlas-based approach and the evaluation scheme used to assess PM segmentation.

\subsection{Materials}

Twenty-three 3D ECG- and respiratory-gated MRI volumes, images size $256 \times$ $256 \times 140$, were acquired at King's College London [11]. We denote this the set $S_{1}$. Two additional 3D Whole Heart ECG- and respiratory-gated MRI datasets, images size $152 \times 256 \times 120$ and $128 \times 256 \times 96$, were acquired at Great Ormond Street Hospital with a different scanner (1.5 Tesla Siemens Avanto). We denote this the set $S_{2}$.

\subsection{Method}

We make use of a multi-atlas based segmentation approach. In the following, we describe the atlas creation, the selected atlas segmentation method and the evaluation scheme.

Atlas creation. For each scan from $S_{1}$, a manual segmentation of the four main chambers, the myocardium, the pulmonary artery and the aorta was available. ITK-SNAP [12] was adopted to add the labels of the antero-lateral (APM) and postero-medial (PPM) papillary muscles. To ease the labeling task, an initial segmentation was obtained with the Snake toolbox. The region of interest was selected around each muscle and a thresholding filter applied to correctly drive the active contour evolution. One or more balloons were placed within each muscle main region and the snake evolution performed until convergence imposing expanding balloon force and low curvature constraint. Manual editing was performed in the obtained result to correct for errors caused by the irregular and branched shape of the papillaries. 
Using the available labels for $S_{1}$ two different atlas were created. An atlas set containing all the possible available labels (whole heart and papillary muscles), which we denote WHPMA, and an atlas set containing only the labels of the PM. We denoted this atlas set $P M A$. Set $S_{2}$ was not included in the atlas and was only used for validation purposes.

Multi-Atlas Segmentation. There is a wide range of multi-atlas based approaches addressing the problem of cardiac segmentation [7]. We have selected to use the segmentation pipeline proposed by Zuluaga et al. [10] as it has shown to be robust in the segmentation of different structures within the heart [13, 14]. The details of the presented method can be found in [10]. Here we only give a brief description of it.

Let an atlas database $A$ be expressed as the set of $n$ paired images $A=$ $\left\{Y_{j} ; L_{j}\right\}, j \in\{1, \ldots, n\}$, with $Y_{j}$ an intensity image and $L_{j}$ a label image, and let $Y_{u}$ be an unseen image to be diagnosed. A segmentation for $Y_{u}$ is obtained by transforming the set of $n$ atlases into the image space of $Y_{u}$ and then applying a fusion criterion to combine the label images $L_{j}$ from each atlas into a consensus segmentation $\hat{L}_{u}$. To determine whether an unseen image $Y_{u}$ contains a specific pathology, it has to be segmented using an atlas set with the same pathological pattern.

The structures surrounding the heart tend to bias the registration in cardiac images. To avoid this problem, the atlas set $A$ is registered to $Y_{u}$ in a two-stage process. In the first stage, a region of interest that encloses the heart is obtained by affinely registering $Y_{u}$ to every $Y_{j}$, applying the obtained transformations to binarised $L_{j}$ images, and finally fusing those into a mask $M_{u}$ using a majority voting criterion. In the second stage, this mask is applied to the unseen image to allow for flexible registrations without bias. With a nonrigid free form deformation registration using normalised mutual information, we align the entire atlas set $A$ to $Y_{u}$.

The final segmentation $\hat{L}_{u}$ is obtained by using the multi-STEPS algorithm [15] in combination with a locally normalised cross correlation (LNCC) based ranking strategy to determine which are the most suitable atlases to use in the fusion process. The STEPS algorithm provides a parameter $X$ that allows one to control the number of atlases to use locally according to the LNCC.

Evaluation Scheme The capability of the multi-atlas segmentation method to obtain a satisfactory extraction of the PM was evaluated with using leaveone-out cross validation scheme on set $S_{1}$, i.e. each image was automatically segmented adopting the remaining twenty-two ones as atlas. Two different tests were performed: one using WHPMA, another one using PMA.

The obtained results were compared with the manual segmentation in terms of visual assessment and Dice score, the latter computed as

$$
\text { Dice }=\frac{2\left(V_{\text {manual }} \cap V_{\text {automatic }}\right)}{\left(V_{\text {manual }} \cup V_{\text {automatic }}\right)}
$$


Additionally, the PM's from $S_{2}$ were segmented with $W H P M A$ and $P M A$ using the complete set (23 images) and visually assessed. The goal of this experiment was to determine the sensibility of the method to different image scanners.

\section{$3 \quad$ Validation and Results}

Table 1 shows the results of computing the Dice scores for both the APM and PPM segmentations in $S_{1}$. The values were averaged between the twenty-three datasets and the standard deviation was computed. A reduction of the mean Dice scores is noticeable when the atlas adopted had only the papillary muscles labels.

Table 1. Mean, standard deviation (St Dev), maximum (Max) and minimum (Min) Dice scores computed between manual and multi-atlas segmentation for each image of the atlas. Results are shown when adopting the completely labelled atlas WHPMA and the one with only the papillaries labels $P M A$.

\begin{tabular}{c|cc|cc}
\hline \hline Dice & WHPMA & APM & PHA & PPM \\
& 0.60 & 0.51 & 0.73 & $P M A$ \\
\hline \hline Mean & 0.14 & 0.19 & 0.06 & 0.71 \\
St Dev & 0.77 & 0.73 & 0.81 & 0.10 \\
Max & 0.22 & 0.00 & 0.62 & 0.83 \\
Min & 0
\end{tabular}

In Fig. 2 the manual segmentation of one image is compared against the one obtained with the multi-atlas approach. The automatic method is able to correctly segment the two papillary muscles in all the tested datasets, although the regions presenting thin and irregular branches remain difficult to label. As a result of the label fusion, the great majority of the obtained segmentation shows papillaries with a more homogeneous and smooth shape than the ground truth ones, hence influencing the values of the Dice scores. The results of adopting the atlas with only the papillary muscles labels $(P M A)$ are presented in Fig. 2, showing a worsening of the segmentation, and suggesting the importance of the surrounding structures to guide through a correct identification of the PM.

Finally, Fig. 3 shows the results of using the proposed fully labelled atlas to segment two unseen datasets acquired with different machines and MRI sequences. One can notice a good identification of all the cardiac structures and of the papillary muscles, giving credit to the robustness of the method in automatically identifying and precisely labelling most of the cardiac structures of interest. 

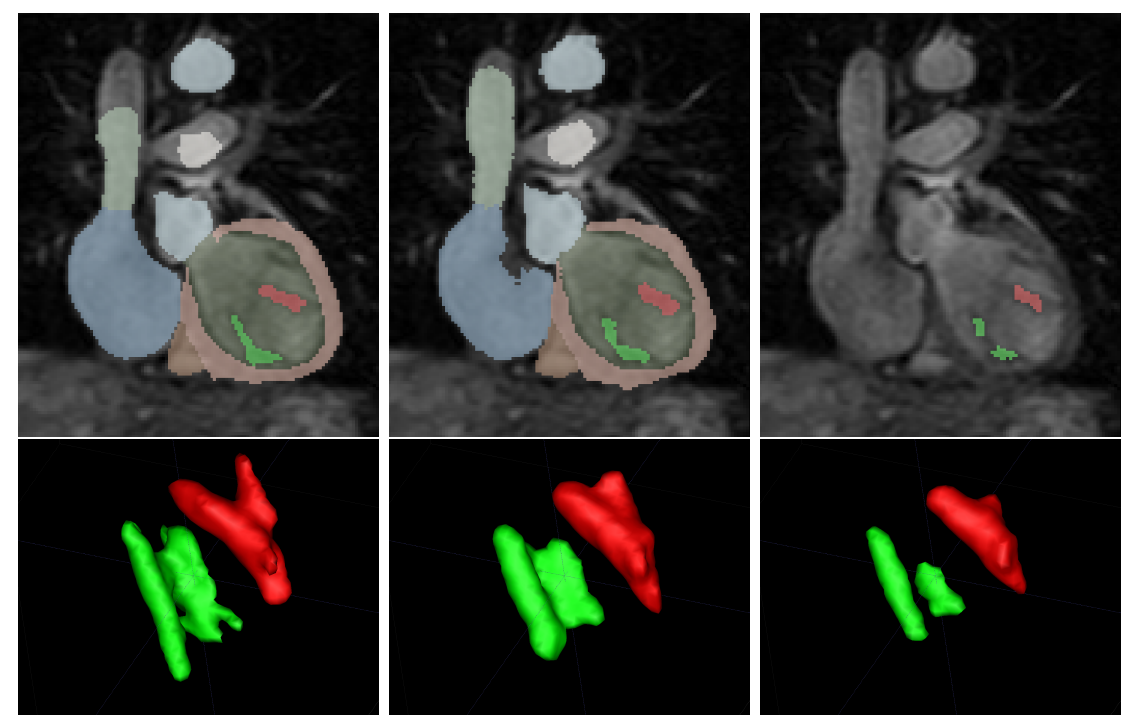

Fig. 2. Coronal views and $3 \mathrm{D}$ rendering of the whole heart segmentation, with particular focus on APM (green) and PPM (red). a) Ground truth manual segmentation. b) Automatic segmentation obtained with the fully labelled atlas. c) Automatic segmentation obtained with the atlas where only the papillary muscles labels were provided.

\section{Discussion and Conclusions}

In this work we have presented the usage of a whole heart multi-atlas segmentation framework for the extraction of the papillary muscles from MR images. Despite the popularity of atlas-based methods in cardiac imaging, no previous work had attempted the segmentation of the PM under this framework. Although the PM have an irregular, branched shape and their identification within the LV is strictly influenced by the image quality and contrast, we demonstrated that an atlas-based segmentation method represents a robust and feasible strategy for their identification and labelling. The obtained results perform better than a previous approach using the same image modality [8].

We evaluated two different atlas sets: one containing only the PM and another one containing labels for other structures of the heart. The results suggest that the use of extra labels improves the quality of the segmentation. Further improvements of the segmentation could be achieved if an extra refinement step could be applied to the results, as proposed in [9]. However, the obtained quality is sufficient enough for our final aim. Furthermore, it is preferable to have one single segmentation framework rather than a complex pipeline connecting different methods.

The main challenges encountered were the correct classification of the PM with respect to the LV, and the precise representation of the branched structure of the muscles. In order to improve the quality of the results, one could extend the 

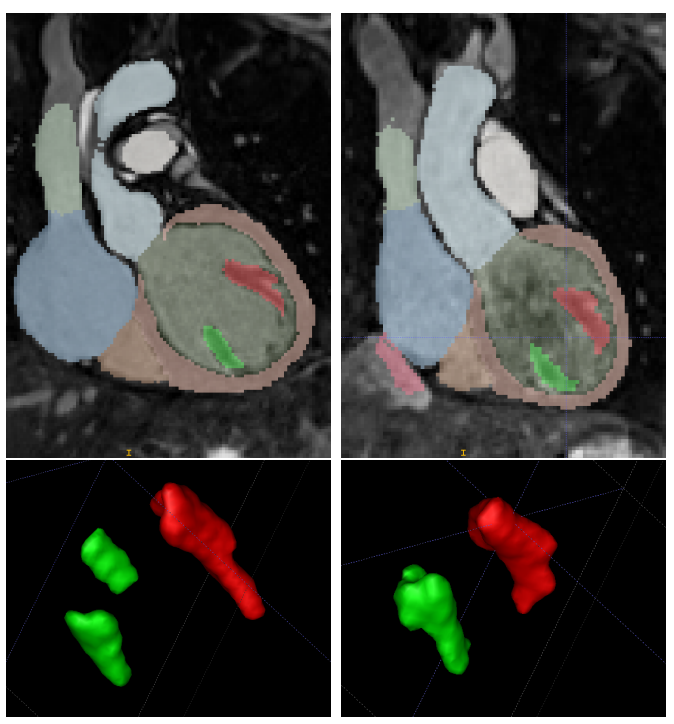

Fig. 3. Result of applying the automatic segmentation method with the proposed atlas on two new datasets acquired with different scanner and MRI sequences. Coronal view and $3 \mathrm{D}$ rendering of the papillary muscles segmentation.

algorithm with a further step, i.e the segmentation of the papillaries alone can be performed after masking the surrounding structures with a mask corresponding to the LV, obtained with a preliminary full-heart segmentation. Nevertheless, we believe that the quality of the segmentation obtained within this work is sufficient for our scope, i.e. automatic landmark detection. In conclusion, the adoption of this method for the segmentation of the papillary muscles in MRI images is the first key step for the development of a multi-modality fusion method able to combine MRI and 3D TTE of the mitral valve apparatus. To the best of our knowledge, this is the first time that a fully automated segmentation method is succesfully applied to MRI for the extraction of the papillary muscles.

Acknowledgments. BB is funded by BLA BLA BLA. SO receives funding from the EPSRC (EP/ H046410/1, EP/J020990 / 1, EP/K005278), the MRC (MR/ J01107X/1), the EU-FP7 project VPH-DARE@ IT (FP7-ICT-2011-9-601055), the NIHR Biomedical Research Unit (Dementia) at UCL and the National Institute for Health Research University College London Hospitals Biomedical Research Centre (NIHR BRC UCLH/UCL High Impact Initiative). AMT and SS are funded by BLA BLA BLA

\section{References}

1. McCarthy, K.P., Ring, L., Rana, B.S.: Anatomy of the mitral valve: understanding the mitral valve complex in mitral regurgitation. European Heart Journal - 
Cardiovascular Imaging 11(10) (2010) i3-i9

2. Reinertsen, I., Descoteaux, M., Siddiqi, K., Collins, D.: Validation of Vessel-based Registration for Correction of Brain Shift. Medical Image Analysis 11 (2007) 374-388

3. Rivaz, H., Chen, S.S., Collins, D.: Automatic deformable mr-ultrasound registration for image-guided neurosurgery. Medical Imaging, IEEE Transactions on 34(2) (Feb 2015) 366-380

4. Huang, X., Hill, N.A., Ren, J., Guiraudon, G., Boughner, D., Peters, T.M.: Dynamic $3 \mathrm{~d}$ ultrasound and $\mathrm{mr}$ image registration of the beating heart. In: Medical Image Computing and Computer-Assisted Interventions -MICCAI. (2005) 171-178

5. Huang, X., Hill, N., J, R., T.M., P.: Rapid registration of multimodal images using a reduced number of voxels procedures. In: SPIE Medical Imaging. Volume 6141. (2006) 34756

6. Ma, Y.L., Penney, G.P., Rinaldi, C.A., Cooklin, M., Razavi, R., Rhode, K.S.: Echocardiography to magnetic resonance image registration for use in image-guided cardiac catheterization procedures. Physics in Medicine and Biology 54 (2009) $5039-5055$

7. Zhuang, X.: Challenges and methodologies of fully automatic whole heart segmentation: A review. Journal of Healthcare Engineering (3) (2013) 371-407

8. Spreeuwers, L., Bangma, S., Meerwaldt, R., Vonken, E.and Breeuwer, M.: Detection of trabeculae and papillary muscles in cardiac $\mathrm{mr}$ images. In: Computers in Cardiology. (2005) 415-418

9. Gao, M., Chen, C., Zhang, S., Qian, Z., Metaxas, D., Axel, L.: Segmenting the papillary muscles and the trabeculae from high resolution cardiac ct through restoration of topological handles. In: IPMI. Volume 7917 of LNCS. (2013) 184-195

10. Zuluaga, M., Cardoso, M., Modat, M., Ourselin, S.: Multi-atlas propagation whole heart segmentation from mri and cta using a local normalised correlation coefficient criterion. In: Functional Imaging and Modeling of the Heart (FIMH). Volume 7945 of LNCS. (2013) 174-181

11. Uribe, S., Tangchaoren, T., Parish, V., Wolf, I., Razavi, R., Greil, G., Schaeffter, T.: Volumetric cardiac quantification by using $3 \mathrm{~d}$ dual-phase whole-heart mr imaging1. Radiology 248 (2008) 606-614

12. Yushkevich, P.A., Piven, J., Cody Hazlett, H., Gimpel Smith, R., Ho, S., Gee, J.C., Gerig, G.: User-guided 3D active contour segmentation of anatomical structures: Significantly improved efficiency and reliability. Neuroimage 31(3) (2006) 11161128

13. Petitjean, C., Zuluaga, M.A., Bai, W., Dacher, J.N., Grosgeorge, D., Caudron, J., Ruan, S., Ayed, I.B., Cardoso, M.J., Chen, H.C., Jimenez-Carretero, D., LedesmaCarbayo, M.J., Davatzikos, C., Doshi, J., Erus, G., Maier, O.M., Nambakhsh, C.M., Ou, Y., Ourselin, S., Peng, C.W., Peters, N.S., Peters, T.M., Rajchl, M., Rueckert, D., Santos, A., Shi, W., Wang, C.W., Wang, H., Yuan, J.: Right ventricle segmentation from cardiac mri: A collation study. Medical Image Analysis 19(1) (2015) $187-202$

14. Tobón-Gómez, C., Geers, A., Peters, J., Weese, J., Pinto, K., Karim, R., Ammar, M., Daoudi, A., Margeta, J., Sandoval, Z., Stender, B., Y. Zheng, M.Z.J.B., Ayache, N., Chikh, M.A., Dillenseger, J.L., Mahmoudi, B.M.K.K.S., Sébastien Ourselin, A.S., Schaeffter, T., Razavi, R., Rhode, K.: Benchmark for algorithms segmenting the left atrium from $3 \mathrm{~d}$ ct and mri datasets. IEEE Transactions on Medical Imaging (2015) 
15. Cardoso, M., Leung, K., Modat, M., Keihaninejad, S., Cash, D., Barnes, J., Fox, N., Ourselin, S.: STEPS: Similarity and Truth Estimation for Propagated Segmentations and its application to hippocampal segmentation and brain parcelation. Medical Image Analysis 17(6) (2013) 671 - 684 